\title{
Efficient $\mathrm{XeF}(C \rightarrow A)$ Laser Excited by a Coaxial Electron Beam at Intermediate Pumping Rates
}

\author{
PETER J. PETERS, HERBERT M. J. BASTIAENS, W. J. WITTEMAN, \\ ROLAND SAUERBREY, MEMBER, IEEE, C. BRENT DANE, AND
}

FRANK K. TITTEL, FELLOW, IEEE

\begin{abstract}
$A$ bstract-XeF $(C \rightarrow A)$ laser radiation has been produced in a fivecomponent gas mixture excited by a coaxial electron beam with an intermediate pulse length $(-175 \mathrm{~ns})$ and pumping rate $\left(\sim 1 \mathrm{MW} / \mathrm{cm}^{3}\right)$. The laser output energy was $215 \mathrm{~mJ}$ from an active volume of $157 \mathrm{~cm}^{3}$ $(1.4 \mathrm{~J} / \mathrm{L})$ at a total gas pressure of 8 bar corresponding to an intrinsic efficiency of $0.3 \%$.
\end{abstract}

\section{INTRODUCTION}

$\mathrm{T}$ HE first electron beam or discharge excited XeF lasers operated on the $351 \mathrm{~nm} \mathrm{XeF}(B \rightarrow X)$ transition of the $\mathrm{XeF}$ molecule because the cross section for stimulated emission for this transition is more than one order of magnitude larger than the one for the $\operatorname{XeF}(C \rightarrow A)$ transition. The $\operatorname{XeF}(C \rightarrow A)$ transition is characterized by a broad-band emission spectrum centered around 480 $\mathrm{nm}$ in the blue-green spectral region. Early demonstrations of laser emission from this transition used threecomponent gas mixtures as is common for most excimer systems, which resulted in a relatively inefficient $\mathrm{XeF}(C$ $\rightarrow A$ ) laser. Since 1983, however, significant progress has been made by Nighan et al. using a superior five-component gas mixture [1], [2]. With these improvements the $\mathrm{XeF}(C \rightarrow A)$ laser performance approaches that of the $B$ $\rightarrow X$ excimer system. The kinetic properties were also studied in detail by Nighan et al. [3] and the predicted results agreed well with the experimental results, indicating that the major formation and absorption processes are reasonably well understood.

In most investigations concerning the $\operatorname{XeF}(C \rightarrow A)$ laser, short pulse, high current density electron beams have been used to excite the laser gas. Injection control of these systems has been used to tune the laser wavelength and to narrow the bandwidth, and to significantly enhance the laser output energy to the $\sim 1 \mathrm{~J}$ level with a specific energy of $1.5 \mathrm{~J} / \mathrm{L}$ [4], [5]. On the other hand, recently an electron beam pulse of 700 ns pulse duration

Manuscript received November 17, 1989; revised February 23, 1990 This work was supported by the Dutch Stichting FOM, the Office of Naval Research, the National Science Foundation, and the Welch Foundation.

P. J. Peters, H. M. J. Bastiaens, and W. J. Witteman are with the Department of Applied Physics, Univeristy of Twente, 7500 AE Enschede, The Netherlands.

R. Sauerbrey, C. B. Dane, and F. K. Tittel are with the Department of Electrical and Computer Engineering, Rice University, Houston, TX 77251 .

IEEE Log Number 9037365 with a moderate pump rate of $250 \mathrm{kWcm}^{-3}$ was used by Mandl et al. [6] to excite the same five-components laser gas mixture, previously shown to yield optimal results by Nighan et al. [2]. A $\mathrm{XeF}(C \rightarrow A)$ laser pulse width of $400 \mathrm{~ns}$ and a specific energy of $1.25 \mathrm{~J} / \mathrm{L}$ were reported under low-pressure (1.6 atm) excitation conditions [6]. For short $(\sim 10 \mathrm{~ns})$ excitation pulses, the $\operatorname{XeF}(C \rightarrow A)$ operates after termination of the pumping pulse in the afterglow and efficient energy extraction results from injection control. In the long pumping pulse quasi-CW regime the $\mathrm{XeF}(C \rightarrow A)$ gain is very low $\left(\sim 0.005 \mathrm{~cm}^{-1}\right)$ which leads to long laser ring up times $(\sim 300 \mathrm{~ns})$ in a stable cavity [6]. Kinetic modeling indicates that an intermediate pumping regime with excitation pulse length on the order $\sim 100 \mathrm{~ns}$ and pumping powers of about $1 \mathrm{MWcm}^{-3}$ may offer an interesting compromise between the short pulse and long pulse pumping conditions considered earlier [3]. Therefore, in this paper we describe experiments carried out with an electron-beam apparatus that produced pump pulses with an intermediate pulse length of about $175 \mathrm{~ns}$ (FWHM) and a power deposition rate on the order of $1 \mathrm{MWcm}^{3}$. Moreover, we will present initial measurements with gas mixtures in which part of the Ar is replaced by $\mathrm{Ne}$ as a buffer gas. The lower stopping power of $\mathrm{Ne}$ could be compensated for by using a higher gas pressure because of the fact that our system could be pressurized to a total gas pressure of 10 bar. It was found in earlier experiments with other excimers $(\mathrm{ArF}, \mathrm{KrF})$ that the variation of the buffer gas may have important consequences on the optimum pressure regime, total output energy, and kinetics [7]. Moreover, useful information can be gained for discharge excited systems, although the ionic channel dominates the electron-beam excitation $\mathrm{ki}$ netics.

\section{ExPERIMENTAL CONFIgURATION}

To excite the laser gas, a five-stage Marx generator connected directly to a coaxially-shaped vacuum diode was used. The anode was a thin-walled $(50 \mu \mathrm{m})$ titanium tube with an inner diameter of $20 \mathrm{~mm}$ which contained the laser gas mixture. The construction of the tube allowed the system to be pressurized up to 10 bar for several hundreds of shots without a tube failure. The cathode was a cylindrical metal tube with an inner diameter of $128 \mathrm{~mm}$. 


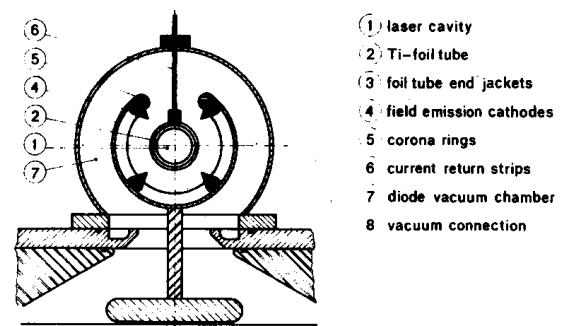

(a)

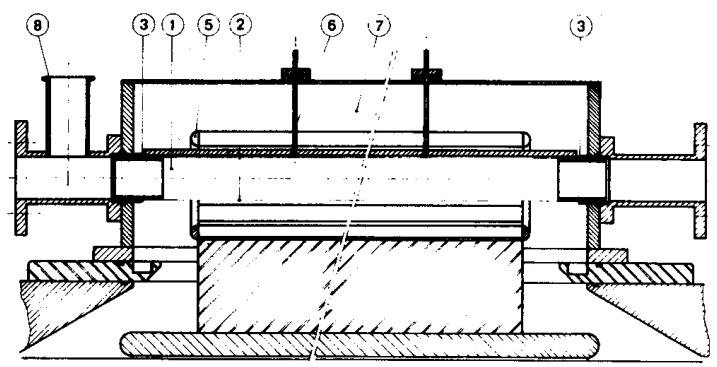

(b)

Fig. 1. Transverse (a) and longitudinal (b) cross section of the coaxial electron beam diode and the laser chamber.

On the innerside four solid graphite edge-shaped profiles each of $50 \mathrm{~cm}$ length were mounted. Cross sections of the coaxial diode structure are shown in Fig. 1. The apparatus is described in detail in [8]. The pulse length of the electron beam in the diode was about $250 \mathrm{~ns}$ (FWHM) at a maximum peak diode voltage of $275 \mathrm{kV}$ and peak current of $27.5 \mathrm{kA}$. The estimated peak current density in the laser cell was about $100 \mathrm{~A} / \mathrm{cm}^{2}$. This was confirmed by the power deposition calculations from the stopping power tables which agreed with the values obtained from the energy deposition measurements by the pressure jump technique. Due to the stopping power of the titanium tube wall, the laser current pulse width inside the anode tube is about $175 \mathrm{~ns}$ (FWHM). This was concluded from fluorescence measurements on this device. An example of such a fluorescence signal of the $\operatorname{XeF}(B \rightarrow X)$ transition is shown in Fig. 4.

The optical cavity was formed by two dielectric mirrors separated by $80 \mathrm{~cm}$. The radius of curvature of the first mirror was $10 \mathrm{~m}$, with a reflectivity of $99.8 \%$ at $485 \pm$ $20 \mathrm{~nm}$ and a transmission of more than $90 \%$ at $350 \mathrm{~nm}$. The other mirror was plane-plane and was coated with a dielectric stack with a reflectivity of $85 \%$ at $485 \pm 20 \mathrm{~nm}$ and a transmission of more than $90 \%$ at $350 \mathrm{~nm}$. All optics were protected by an $\mathrm{Al}_{2} \mathrm{O}_{3}$ overlay to minimize fluorine damage of the dielectric coatings.

The laser output beam passed through a color glass filter (GG 385) and was detected by a GenTec ED 500 energy meter. The reflected signal from the color glass filter was used to monitor the temporal shape of the output pulse. It was detected after appropriate attenuation by

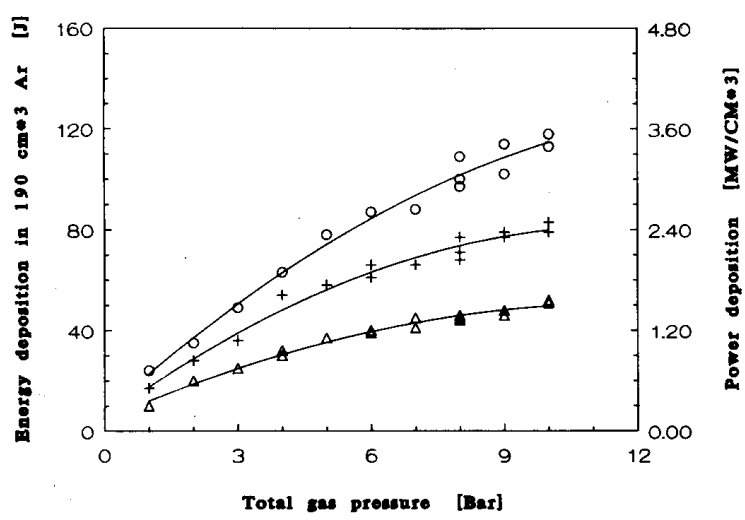

Fig. 2. Energy deposition as a function of the total gas pressure for various load voltages of the Marx generator $(\Delta: 80 \mathrm{kV} ;+: 90 \mathrm{kV}, \mathrm{O}: 100 \mathrm{kV}$; volume: $190 \mathrm{~cm}^{3}$; gas mixture $3 \% \mathrm{HCl}$ and $97 \% \mathrm{Ar}$ ).

neutral density filters, a diffuser, and a $485 \pm 10 \mathrm{~nm}$ broad-band interference filter by a vacuum photodiode (ITL TF 1850).

\section{Experimental Results and Discussion}

In order to estimate the intrinsic efficiency, the energy deposition of the electron beam in the gas mixture contained in the laser cell was determined. In Fig. 2 the results are shown for different load voltages of the Marx generator as measured by the pressure jump technique. The values depicted have been obtained with a gas mixture containing $3 \% \mathrm{HCl}$ and $97 \% \mathrm{Ar} . \mathrm{HCl}$ was added in order to suppress energy loss through $\mathrm{Ar}_{2}^{*}$ emission. The peak energy depositions are reached at the maximum pressue of 10 bar and correspond to 605,420 , and 220 $\mathrm{J} / \mathrm{L}$ for charging voltages of 100,90 , and $80 \mathrm{kV}$ respectively. At $8 \mathrm{~atm}$ Ar and $100 \mathrm{kV}$ charging voltage the deposited energy is $540 \mathrm{~J} / \mathrm{L}$, which corresponds to pumping power of about $3.1 \mathrm{MWcm}^{-3}$.

Previous experiments had shown that a $6 \% \mathrm{Kr}$ concentration in the laser gas mixture is optimum for maximum energy output from the $\operatorname{XeF}(C \rightarrow A)$ laser [2]. Recent experiments with short electron beam pulse excitation suggested that a higher $\mathrm{Kr}$ concentration of about $25 \%$ might be preferable [5]. Initial measurements of the laser output energy under the present pumping conditions showed that a five-component laser gas mixture containing $24 \%$ of $\mathrm{Kr}$ resulted in lower laser output levels compared to a mixture containing $6.1 \%$ of Kr. In Fig. 3 the output energy is plotted as a function of the total gas pressure containing $0.02 \% \mathrm{~F}_{2}, 0.16 \% \mathrm{NF}_{3}, 0.18 \% \mathrm{Xe}, 6.1 \%$ $\mathrm{Kr}$, and $93.5 \%$ Ar. The maximum output energy of 215 $\mathrm{mJ}(1.4 \mathrm{~J} / \mathrm{L}$, intrinsic efficiency: $0.26 \%)$ was obtained at a total gas pressure of 8 bar. For a higher gas pressure the output energy decreased although the pump rate increased further. For the lower pump rate $(90 \mathrm{kV}$ Marx load voltage) the output energy began to saturate at a total gas pressure of 7 bar. Energy measurements with and without the color glass filter showed no detectable emission at 


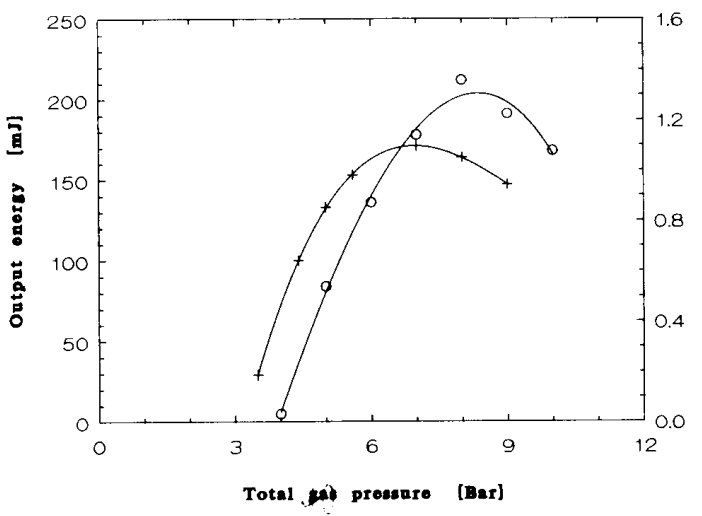

Fig. 3. $\mathrm{XeF}(C \rightarrow A)$ output energy as a function of the total gas pressure for two different load voltages $(+: 90 \mathrm{kV}, 0: 100 \mathrm{kV}$; active volume: $\left.157 \mathrm{~cm}^{3}\right)$.

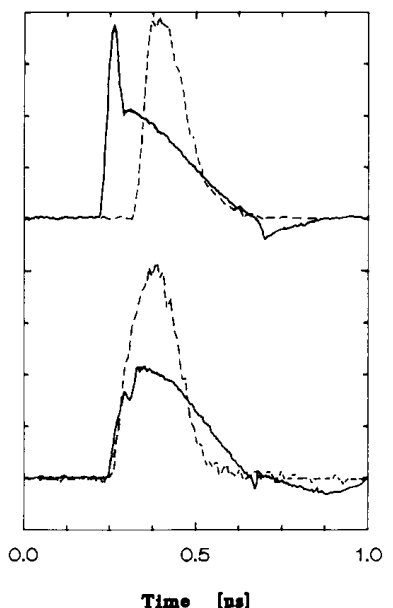

Fig. 4. Upper part: Electron beam diode voltage (solid line) and temporal development of the laser output pulse (dashed line). Lower part: Current pulse (solid line) and $\operatorname{XeF}(C \rightarrow A)$ fluorescence signal (dashed line); charging voltage $90 \mathrm{kV}$; total gas pressure: 5 bar.

either $351 \mathrm{~nm}$, the $\mathrm{XeF}(B \rightarrow X)$ transition, or the $\mathrm{KrF}$ transition at $248 \mathrm{~nm}$. There is some spread in the output energy values measured under the same conditions. For this reason, a fresh gas mixture was used for each shot.

In Fig. 4, a typical set of time dependent signals is plotted (charging voltage: $90 \mathrm{kV}$; total gas pressure: 5 bar). In the upper part of the figure the voltage is shown (solid line) together with the laser output pulse (dashed line). In the lower part the current (solid line) and a fluorescence signal (dashed line) are plotted as a function of time. The fluorescence signal shown here is a $\operatorname{XeF}(C \rightarrow A)$ signal measured by a photomultiplier with a suitable neutral density and bandpass filter. The signal was measured from a pumped length of $0.5 \mathrm{~cm}$. They started at the same time as the current signal and showed the same width as the $\mathrm{XeF}(B \rightarrow X)$ signals. Fluorescence signals from the entire pumping length showed considerable narrowing to a

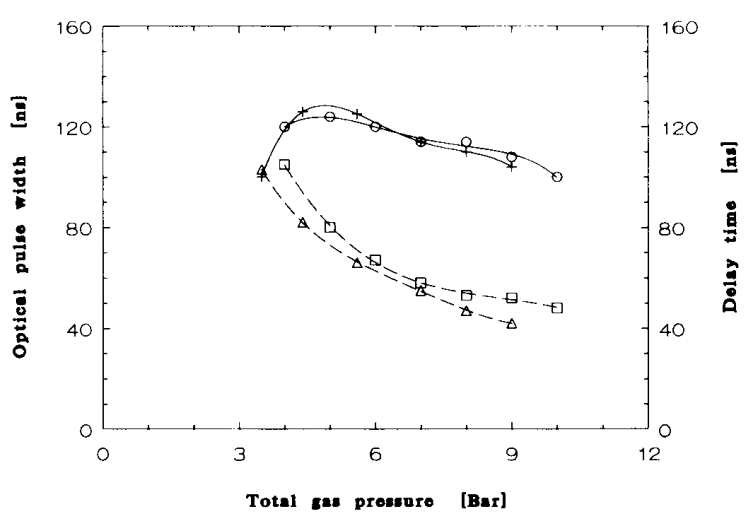

Fig. 5. Optical pulse width (solid line) and delay time (dashed line) as a function of the total laser gas pressure for two different load voltages $(+, \square: 90 \mathrm{kV}, \bigcirc, \Delta: 100 \mathrm{kV})$.

pulse length of $\sim 50 \mathrm{~ns}$ (FWHM) due to amplified spontaneous emission. The delay time or optical ring-up time is the time difference between the onset of the current (and fluorescence signal) and the laser output signal. Fig. 5 shows the delay time and the width of the laser pulses as a function of gas pressure. The delay times show a considerable decrease with increasing gas pressures. At low gas pressures $(-3$ bar) the gain is small due to the low pumping density which causes a relatively long ring-up time of about $95 \mathrm{~ns}$ in the stable resonator. At higher pumping densities, i.e., higher gain coefficients, the ringup time decreases to $40 \mathrm{~ns}$. The temporal laser pulse width is almost pressure independent. The longest pulse of about $130 \mathrm{~ns}$ was seen at a low pressure, while for 10 bar a value of $100 \mathrm{~ns}$ was measured.

Calculations for this system were performed based on an analytical model for injection controlled excimer laser amplifiers [9]. Since the gain coefficient under the present pumping conditions was not known, it was inferred from the laser output energy. This yielded a value of $\sim 0.01$ $\mathrm{cm}^{-1}$ under optimum pumping conditions at 8 bar. If this gain coefficient is assumed the calculated laser pulse width and delay times are in good agreement with the experimental values (Fig. 5). The predicted optimum reflectivity for the output coupler is between $90 \%$ and $95 \%$ rather than at $85 \%$ which was used in anticipation of a higher gain for the present experiments. With optimized cavity conditions energy outputs of $\sim 400 \mathrm{~mJ}$ are predicted.

Experiments were also conducted with the same gas mixture in which $50 \%$ of the Ar buffer gas was replaced by $\mathrm{Ne}$. The results of the measurements with $50 \% \mathrm{Ne}$ are plotted in Figs. 6 and 7. The output energy under these conditions was lower, but there was little change in the shape of the curves. The optical delay times were about 30 ns longer compared to the gas mixtures with only Ar as a buffer gas. A comparison of the pulse widths shows that the pressure dependence for the $\mathrm{Ne}-\mathrm{Ar}$ mixture was smaller than for the Ar buffered mixture. Especially in the low-pressure gas mixtures, the combination of a longer 


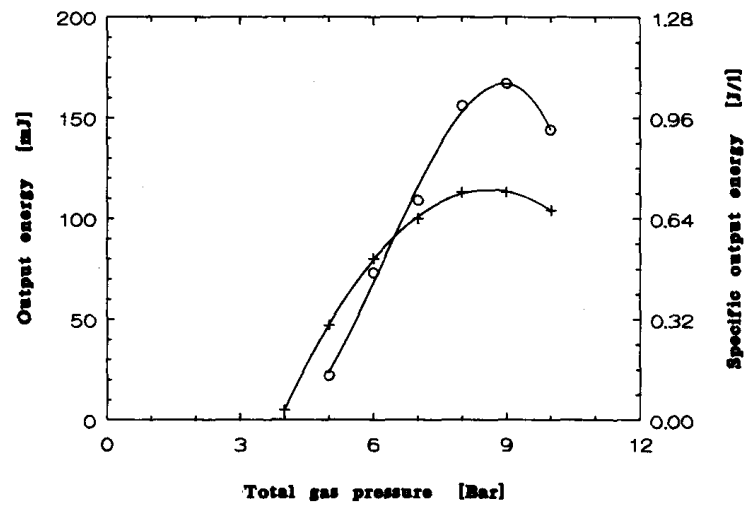

Fig. 6. Same as Fig. 3, but in the laser gas mixture $50 \%$ of the $\mathrm{Ar}$ is replaced by Ne.

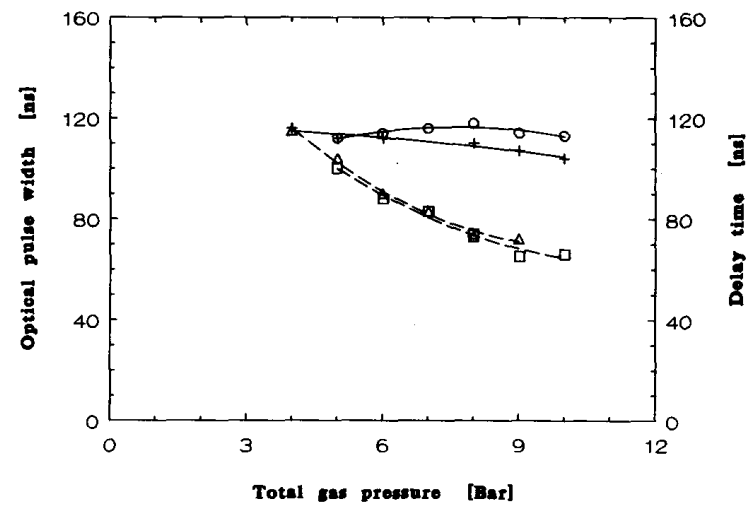

Fig. 7. Same as Fig. 5 with $50 \%$ Ne instead of Ar.

delay time and a shorter pulse width accounted for the lower output energy values. We could not obtain a high laser fluence from a laser gas mixture containing $75 \% \mathrm{Ne}$, probably due to degradation of the optics.

In conclusion, we have shown that the $\operatorname{XeF}(C \rightarrow A)$ laser can also be operated successfully in the intermediate electron beam pumping regime. In order to obtain an output energy of $\sim 1.4 \mathrm{~J} / \mathrm{L}$, it was not necessary to use injection control. On the other hand, with injection control the output energy should increase, especially at low pressure, due to a decrease laser ring-up time. The system was not optimized with respect to the laser gas mixture and the output mirror reflectivity. Moreover, all results have been obtained with a titanium anode tube with a wall thickness of $50 \mu \mathrm{m}$. By changing to a $25 \mu \mathrm{m}$ tube, the pumping rate can be increased considerably and even better results can be achieved with this $\operatorname{XeF}(C \rightarrow A)$ excimer laser system.

\section{REFERENCES}

[1] W. L. Nighan, Y. Nachshon, F. K. Tittel, and W. L. Wilson, "Optimization of electrically excited $\operatorname{XeF}(C \rightarrow A)$ laser performance," Appl. Phys. Lett., vol. 42, p. 1006, 1983.
[2] W. L. Nighan, F. K. Tittel, W. L. Wilson, N. Nishida, Y. Zhu, and R. Sauerbrey, "Synthesis of rare gas halide mixtures resulting in efficient $\operatorname{XeF}(C \rightarrow A)$ laser oscillation," Appl. Phys. Lett., vol. 45, p. 947, 1984.

[3] W. L. Nighan and M. C. Fowler, "Kinetic processes in electron beam excited $\operatorname{XeF}(C \rightarrow A)$ laser media," IEEE J. Quantum Electron., vol. 25, p. 791, 1989.

[4] N. Hamada, R. Sauerbrey, W. L. Wilson, F. K. Tittel, and W. L. Nighan, "Performance characteristics of an injection controlled electron beam pumped $\operatorname{XeF}(C \rightarrow A)$ laser system," IEEE $J$ Quantum Electron., vol. 24, p. $1571,1988$.

[5] G. J. Hirst, C. B. Dane, W. L. Wilson, R. Sauerbrey, F. K. Tittel, and W. L. Nighan, "Scaling of an injection-controlled $\operatorname{XeF}(C \rightarrow A)$ laser pumped by a repetitively pulsed, high current density electron beam," Appl. Phys. Lett., vol. 54, p. 1851, 1989.

[6] A. Mandl and L. H. Litzenberger, "Efficient, long pulse $\mathrm{XeF}(C \rightarrow A)$ laser at moderate electron beam pump rate," Appl. Phys. Lett., vol. 53, p. $1690,1988$.

[7] P. J. M. Peters, I. H. T. Fierkens, and W. J. Witteman, "Effect of Ne and $\mathrm{Ar}$ on the performance of a high pressure $\mathrm{ArF}^{*}$ laser pumped by a small coaxial electron beam," Appl. Phys. Lett., vol. 51, p. 883, 1987.

[8] P. J. M. Peters, H. M. J. Bastiaens, and W. J. Witteman, "A study of the electron quenching of excimers in a $\mathrm{KrF}^{*}$ laser excited by a coaxial electron beam,"' Appl. Phys., vol. B-43, p. 253, 1987.

[9] N. Hamada, R. Sauerbrey, and F. K. Tittel, "Analytical model for injection-controlled excimer laser amplifiers," IEEE J. Quantum Electron., vol. 24, p. 2458, 1988.

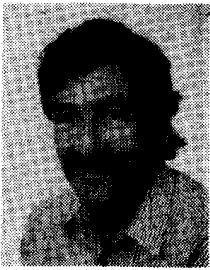

Peter J. Peters was born in Meerlo, The Netherlands on November 5, 1945. He received the M.S. degree from the Catholic University of Nijmegen in 1975 and the Ph.D. degree in 1981 form the University of Twente, Enschede, The Netherlands, all in physics.

In 1981 he joined the staff of the Quantum Electronics Group. He is now an Associate Professor with the University of Twente and has conducted research on TEA CO discharge lasers, various excimer lasers, and atomic rare-gas lasers.

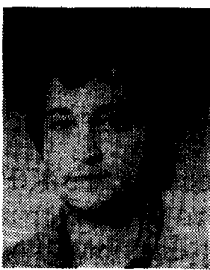

Herbert M. J. Bastiaens was born in Rimburg, The Netherlands on August 31, 1959. He received the diploma in technical physical engineering in 1981.

Since 1981 he has been with the Quantum Electronics Group, Faculty of Applied Physics, University of Twente, Enschede, The Netherlands, where he has been involved in research on electron beam pumped gas lasers.

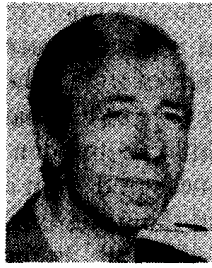

W. J. Witteman was born in Monster, The Netherlands, on December 12, 1933. He received the degree of Mechanical Engineer from the Technical University of Delft, Delft, The Netherlands, in 1958 , the Ph.D. degree in physics from the Technical University of Eindhoven, Eindhoven, The Netherlands in 1963.

He was Postdoctoral Fellow from 1958 to 1959 and a Research Associate from 1959 to 1960 with the Institute for Fluid Dynamics and Applied Mathematics, University of Maryland, College Park, MD, where he was engaged in shock tube research and molecular relaxation phenomena. From 1961 to 1969 he was with Philips Research 
ries, Eindhoven, The Netherlands, and participated in high-pressure physics and laser physics. Since 1969 he has been a professor with the University of Twente in laser physics, where he is currently engaged in highpower molecular lasers $\left(\mathrm{CO}_{2}\right.$ systems, excimer lasers, electroionizaton lasers) and free-electron lasers.

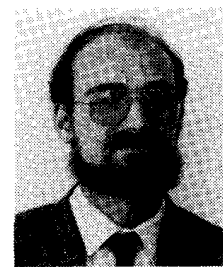

Roland Sauerbrey ( M' 85) was born in Coburg, Germany, in 1952. He received the M.S. and the $\mathrm{Ph} . \mathrm{D}$. degrees, both in physics, from the University of Würzburg, Würzberg, Germany, in 1978 and 1981 , respectively.

After a year as a postdoctoral fellow with Rice University, Houston, TX, he returned to the University of Würzburg, where he became an Assistant Professor with the Department of Physics. In 1985 he joined the faculty of Rice University where he is presently an Associate Professor with the Department of Electrical and Computer Engineering. His primary research activities have been in the areas of short wavelength lasers, excimer spectroscopy, and laser applications.

Dr. Sauerbrey is a member of the American Optical Society, the American Physical Society and the German Physical Society.

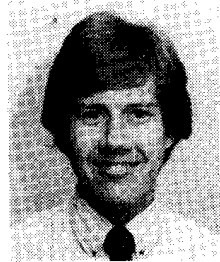

C. Brent Dane was born in Dalhart, TX, on August 8,1961 . He received the B.S. degree in chemistry and mathematics from Wayland Baptist University, Plainview, TX, in 1983 and the M.A and $\mathrm{Ph} . \mathrm{D}$. degrees in physical chemistry from Rice University, Houston, TX, in 1986 and 1987, respectively.

From January 1988 to May 1990 , he was a research associate with the Department of Electrical and Computer Engineering, Rice University. He is presently a staff physicist in the Laser Programs

Division at Lawrence Livermore National Laboratories, Livermore, CA. His primary research activites have been in the development of tunable laser systems in the visible and infrared and their applications in spectroscopy and the study of chemical kinetics.

Dr. Dane is a member of Optical Society of America and Sigma Xi.

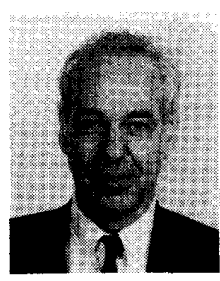

Frank K. Tittel ( SM'72-F'86) was born in Berlin, Germany, in 1933. He received the M.A. and Ph.D. degrees from Oxford University, Oxford, England.

From 1959 to 1967 he was a Research Physicist with the General Electric Research and Development Center, Schenectady, NY. Since 1967 he has been with Rice University, Houston, TX where he is a Professor with the Department of Electrical and Computer Engineering. His research interests include laser devices, laser spectroscopy, and nonlinear optics.

Dr. Tittel is a Fellow of the Optical Society of America and a member of the IEEE Laser and Electro-Optics Society and the American Physical Society. 